\title{
Electrophysiological correlates of impaired reading in dyslexic pre-adolescent children
}

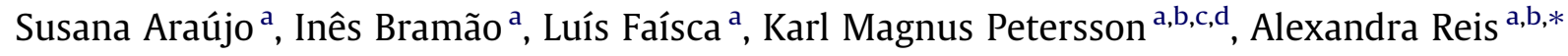 \\ ${ }^{a}$ Cognitive Neuroscience Research Group, Departamento de Psicologia, Institute of Biotechnology Er Bioengineering, Centre for Molecular \\ and Structural Biomedicine, Universidade do Algarve, Faro, Portugal \\ ${ }^{\mathrm{b}}$ Cognitive Neurophysiology Research Group, Stockholm Brain Institute, Karolinska Institutet, Stockholm, Sweden \\ ${ }^{\mathrm{c}}$ Max Planck Institute for Psycholinguistics, Nijmegen, The Netherlands \\ ${ }^{\mathrm{d}}$ Donders Institute for Brain, Cognition and Behaviour, Radboud University, Nijmegen, The Netherlands
}

\section{A R T I C L E I N F O}

\section{Article history:}

Accepted 2 February 2012

\section{Keywords:}

Dyslexia

ERP

Orthographic processing

Phonological processing

\begin{abstract}
A B S T R A C T
In this study, event related potentials (ERPs) were used to investigate the extent to which dyslexics (aged 9-13 years) differ from normally reading controls in early ERPs, which reflect prelexical orthographic processing, and in late ERPs, which reflect implicit phonological processing. The participants performed an implicit reading task, which was manipulated in terms of letter-specific processing, orthographic familiarity, and phonological structure. Comparing consonant- and symbol sequences, the results showed significant differences in the P1 and N1 waveforms in the control but not in the dyslexic group. The reduced $\mathrm{P} 1$ and $\mathrm{N} 1$ effects in pre-adolescent children with dyslexia suggest a lack of visual specialization for letter-processing. The P1 and N1 components were not sensitive to the familiar vs. less familiar orthographic sequence contrast. The amplitude of the later N320 component was larger for phonologically legal (pseudowords) compared to illegal (consonant sequences) items in both controls and dyslexics. However, the topographic differences showed that the controls were more left-lateralized than the dyslexics. We suggest that the development of the mechanisms that support literacy skills in dyslexics is both delayed and follows a non-normal developmental path. This contributes to the hemispheric differences observed and might reflect a compensatory mechanism in dyslexics.
\end{abstract}

(c) 2012 Elsevier Inc. All rights reserved.

\section{Introduction}

Reading is a complex skill that entails a multi-stage process, including visual feature processing, orthographic and phonological analysis, semantic retrieval, and articulatory processes. Due to advances in brain imaging technologies in recent years, it is now possible to investigate the cortical dynamics of the component processes involved in reading and to provide a neurobiological signature for reading failure, as in developmental dyslexia (Dehaene, 2010). A number of brain imaging studies have provided converging evidence that three important neural systems support reading: one anterior system around the inferior frontal gyrus involved in word analysis (decoding), and two posterior systems, one in the parietotemporal region also involved in word analysis, and the other more inferior in the occipitotemporal region which is responsible for fluent and automatic reading (for an overview, see Price \& Mechelli, 2005; Schlaggar \& McCandliss, 2007;

* Corresponding author. Address: Departamento de Psicologia, Faculdade de Ciências Humanas e Sociais, Campus de Gambelas, Universidade do Algarve, 8005139 Faro, Portugal. Fax: +351 289800067.

E-mail address: aireis@ualg.pt (A. Reis).
Shaywitz \& Shaywitz, 2008; Shaywitz et al., 2002). A recent review and meta-analysis of neuroimaging studies of dyslexia found evidence for an atypical neural organization of the reading process in dyslexics relative to non-impaired readers. This is mostly expressed as an underactivation of the left temporoparietal language regions (posterior aspect of the superior temporal gyrus/sulcus, supramarginal gyrus) and the left occipitotemporal cortex, while the inferior frontal system, related to covert articulatory processes, shows an overactivation (as premotor/motor cortex; Maisog, Einbinder, Flowers, Turkeltaub, \& Eden, 2008; Richlan, Kronbichler, \& Wimmer, 2009).

At present, the dominant explanatory framework for dyslexia is that this disorder is caused by a language-specific deficit within the phonological processing system. The phonological deficit explanation of dyslexia contends that dyslexic readers are unable to process the phonological structure underlying word reading, which includes difficulty in manipulating the basic components of language (i.e., graphemes and phonemes). Specifically, poorly specified phonological word representations give rise to a phonemic awareness deficit which hinders the extraction of graphemephoneme associations on which sublexical reading is dependent (Ramus et al., 2003; Snowling, 2000; Vellutino \& Scanlon, 1987). 
A recent reformulation proposes that the deficit may have less to do with the quality of phonological representations and more with access/accessibility to these representations (Ramus \& Szenkovits, 2008).

In support of the phonological deficit hypothesis, dyslexics perform below average on a variety of experimental tasks that require phonological skills. These include verbal short-term memory (e.g., digit span), phonological awareness (e.g., phoneme deletion and rhyme judgments) or phonological decoding (e.g., pseudoword reading) tasks (Ramus et al., 2003; Shaywitz, 2003; Tijms, 2004; Wagner, Torgesen, \& Rashotte, 1994). Moreover, electrophysiological and brain imaging studies have described different brain activation patterns in dyslexic and non-dyslexic readers during performance of such tasks (Georgiewa et al., 1999, 2002; Rumsey et al., 1997; Shaywitz \& Shaywitz, 2005; Shaywitz et al., 1998; Simos, Breier, Fletcher, Bergman, \& Papanicolaou, 2000). For example, the event related potentials (ERPs) literature on phonological effects tends to report a delayed onset of responses that reflect phonological processing in dyslexic compared to normal readers in the N400 range (e.g., Ackerman, Dykman, \& Oglesby, 1994; Rüsseler, Becker, Johannes, \& Münte, 2007). A relatively early negative potential peaking at about $320 \mathrm{~ms}$ (N320) has been also implicated in phonologic transcription (e.g., Bentin, MouchetantRostaing, Giard, Echallier, \& Pernier, 1999). Recently, Jednoróg and colleagues demonstrated that in a phonological priming task, the dyslexics differentiate from controls in both the phonologically incongruent and congruent conditions, that is, dyslexics exhibited reduced $\mathrm{N} 400$ in the former (less negative-going) and enhanced $\mathrm{N} 400$ in the later (more negative-going). This pattern was taken to indicate that in dyslexia the integration of phonologically related information and the ability to detect deviant stimuli are impaired (Jednoróg, Marchewka, Tacikowski, \& Grabowska, 2010). Further, FMRI studies have indicated that there is a relative underactivation in dyslexics in the left posterior parts of the reading network, including the temporal and temporoparietal regions; these are believed to be central for phonological decoding and integration processes (Georgiewa et al., 2002; Pugh et al., 2000a; Sandak, Mencl, Frost, \& Pugh, 2004; Shaywitz et al., 2001). Yet, these deviant brain responses in dyslexic individuals reflect mainly their difficulties with explicit phonological tasks (e.g., many studies have typically focused on indirect meta-phonological awareness tasks like rhyme judgments) and the way in which phonological information is processed in these tasks is not necessarily the same as under normal perceptual conditions. On-line phonological processing capacities in dyslexics lack further investigation.

Moreover, recent research has suggested that visual-orthographic processing may also characterize dyslexic reading. This hypothesis is supported, for example, by behavioral indices showing dyslexic group performance to be poor in tasks that emphasize lexical orthographic processing (e.g., orthographic judgment tasks, Marinelli, Angelelli, Notarnicola, \& Luzzatti, 2009; orthographic lexical decision tasks, Bergmann \& Wimmer, 2008). Neuroimaging studies have offered further insight, suggesting evidence for a reduced level of automaticity in visual word processing in dyslexia, which is subserved by the left occipitotemporal region (e.g., Cao, Bitan, Chou, Burman, \& Booth, 2006; Maurer et al., 2007, 2011; van der Mark et al., 2009; Wimmer et al., 2010).

The left occipitotemporal region has attracted the attention of reading researchers in the recent years. Development of brain processes within this region that are specifically tuned to recurring properties of a writing system allows, within less than $250 \mathrm{~ms}$, the rapid extraction of linguistic information from sequences of letters (separable from higher-order linguistic properties) and the integration of letters into a perceptual word-form (Brem et al., 2006; Parviainen, Helenius, Poskiparta, Niemi, \& Salmelin, 2006; Tarkiainen, Helenius, Hansen, Cornelissen, \& Salmelin, 1999). The left occipitotemporal region corresponds closely to the visual word form area (VWFA) of Cohen and colleagues (Cohen et al., 2002; McCandliss, Cohen, \& Dehaene, 2003), whose primary function during reading is to "support a form of perceptual expertise for visual word recognition that enables rapid perception of visual words in one's own language" (Schlaggar \& McCandliss, 2007, p. 480). More specifically, recent evidence indicates that at least two levels of VWFA specialization exist: a fast, coarse specialization for print (letter sequences vs. visual control stimuli) and a specialization for processing letter sequences also at the whole-word level, as reflected in VWFA sensitivity to the orthographic familiarity of letter sequences (e.g., Bruno, Zumberge, Manis, Lu, \& Goldman, 2008; van der Mark et al., 2009). A number of FMRI studies have suggested that disruption of this region in dyslexic when compared to normal-reading subjects is evident at both coarse, low-level letter-sequences processing, and, also, dyslexics failed to exhibit a second level of VWFA specialization at the higher whole-word level, that is, increased activity for unfamiliar than familiar word-forms (the so-called orthographic familiarity effect; van der Mark et al., 2009; Wimmer et al., 2010; see also, Cao et al., 2006).

Time-sensitive event related potentials have identified the N1 component that peaks within $150-200 \mathrm{~ms}$ as the strongest index of specialized letter-sequences processing (e.g., Bentin et al., 1999; Maurer, Brem, Bucher, \& Brandeis, 2005; Maurer et al., 2007). In addition, some studies have found similar effects in the preceding P1 ( 50-150 ms; Maurer et al., 2005, 2011). Other orthographic characteristics have been shown to modulate the N1, including word frequency. Higher frequency words generally evoke lower amplitude neural responses (Assadollahi \& Pulvermüller, 2003; Hauk \& Pulvermüller, 2004; Sereno, Rayner, \& Posner, 1998). It has been suggested that this effect reflects facilitated access to lexical information (e.g., Hauk \& Pulvermüller, 2004), though the variable word frequency can also affect later stages of word processing (e.g., Hauk, Davis, \& Pulvermüller, 2008).

While a range of studies demonstrate enhanced and left-lateralized N1 to sequences of letters (vs. control stimuli) in skilled readers, some evidence suggests an absence of this N1 effect in the dyslexic population. For example, Maurer et al. (2007) used ERPs to investigate for the first time how tuning for print develops in young dyslexic children and matched, normally developing, readers. The authors demonstrated that over the course of kindergarten to the second grade, there is an increased activation in normal readers in response to visual words as compared to similar control symbols that occurs around $150-270 \mathrm{~ms}$ (N1 component). In contrast, this was absent in the children who developed dyslexia. The authors concluded that the earliest cortical activation that is specifically related to prelexical orthographic analysis is abnormal in dyslexia. Maurer and colleagues' results contrasted with those from a previous study that failed to report a reduction in the N1 response in dyslexics (Simos et al., 2000). However, in a magnetoencephalographic (MEG) study with dyslexic adults, Helenius and colleagues confirmed a delayed activation in left occipito-temporal areas in dyslexics at the point in time during which letter sequence specific signals first emerge during reading ( $150 \mathrm{~ms}$; Helenius, Tarkiainen, Cornelissen, Hansen, \& Salmelin, 1999). These findings were restricted to the most severe cases, which raises the possibility that at some point during development, some dyslexics are able to attain an appropriate brain sensitivity to text. This is a relevant topic on the basis of the developmental trajectory of print specialization in non-impaired readers (i.e., tuning for print plays an important functional role in the early phase of reading acquisition and follows an inverted non-linear U-shaped developmental time course, probably reflecting a full specialization involving more selective brain processes; Brem et al., 2009; Maurer et al., 2006). An open question is whether impaired print specialization is a 
neural marker of dyslexia at the early stages of reading acquisition but throughout the developmental trajectory it gets reduced.

The aim of the present study was twofold. The first aim was further investigation of the time course of electrophysiological responses during print-specific processing in a sample of dyslexics (9-13 years of age) compared to matched non-impaired readers, to shed light on whether processing at this low-level represents a fundamental deficit. To this end, using an implicit reading task, we compared the ERPs elicited by two stimulus types that differed with respect to the amount of letter-specific processing required, consonant vs. symbol sequences. We focused in the P1 and N1 components, as these are the first visual-evoked components that have been shown to reflect letter-sequence-sensitive activation, as distinct from stimulus nonspecific visual feature activation (within the first $250 \mathrm{~ms}$; e.g., Helenius et al., 1999). We expected non-impaired readers to show a specialization for processing sequences of letters in these latency windows of ERPs (i.e., differential processing of consonant over non-orthographic symbols sequences), but not dyslexic readers, reflecting impaired specialization in the dyslexics. In addition, we aimed to assess further whether early ERP responses in the P1 and N1 ranges can demonstrate sensitivity to the orthographic familiarity at the whole-word level (i.e., visual word-form representations at the lexical-level), assessed by means of lexicality and printed frequency effects. Thus, one included high-frequency words, low-frequency words and pseudowords in this study as a venue to test the effect of familiarity in the early $\mathrm{P} 1$ and N1 peaks. Our hypothesis is that lower activation in response to familiar words (vs. unfamiliar words and pseudowords) would reflect sublexical sensitivity in terms of orthographic familiarity at the whole-word level. On the other hand, if P1/N1 reflects primarily letter recognition, we should not obtain ERP differences as a function of stimulus type.

A second main goal of the present study was to explore on-line phonological processing capacities in dyslexics. Our approach to investigate implicit phonological processing was to compare the ERPs elicited in an implicit reading task with consonant sequences and pseudowords, in the N320 component that is believed to be involved in phonological processes (Bentin et al., 1999; Simon, Bernard, Largy, Lalonde, \& Rebai, 2004). The consonant sequences could not be transformed into a coherent phonological structure (i.e., cannot be transcribed at the phonologic level), whereas for pseudowords the phonological pattern is plausible. This contrast reduces a potential confound of the familiarity effect and better isolates the phonological process. Some results in the literature suggest that phonological recoding plays a primary role for maintaining pronounceable stimuli in working memory (e.g., phonological loop, Baddeley, 1986; Ziegler \& Jacobs, 1995). Conceivably, only pseudowords, but not consonant sequences, can activate phonological information and, thus, benefit from phonological recoding. Based on this, a possible prediction was that, in both reading groups, the N320 should be elicited more extensively by pseudowords than by unpronounceable consonant sequences. Moreover, we expected that reduced amplitudes in response to pseudowords would be shown in the dyslexics compared to controls due to their reduced ability in phonological coding mechanisms (grapheme-tophoneme correspondence, phoneme blending), as well as attenuated differences in N320 ERP amplitudes between pseudowords and consonant sequences for the dyslexics.

\section{Material and methods}

\subsection{Participants}

Twenty pre-adolescent children with dyslexia aged 9-13 years (13 males and 7 females; mean age $[ \pm$ std $]=10.7[ \pm 1.6]$ years) were tested in this study. They were recruited through private clinics specialized for children with special needs and all had been formally diagnosed with dyslexia by a specialized therapist. In this study, the inclusion criteria for the dyslexic participants were as follows: normal-range intelligence measured by the Raven Colored Matrices (Raven, Court, \& Raven, 1998); reading abilities significantly below grade mean level; absence of neurological, emotional, or attentional problems. The individual reading achievement was assessed through the time-limited reading aloud test, adapted for the Portuguese population from the Differential Diagnosis Dyslexia Battery (3DM; Blomert \& Vaessen, 2009). The reading test contained two subtasks of high- and low-frequency word (half a minute for each subtask); reading speed was taken as the number of correct items read per second. Scores were converted into z-scores with reference to normative data, which was collected in a large-scale study with 820 Portuguese children in grades $1-4$ (test-retest reliability $=.91$ ). Only those subjects who had reading speed scores at least 1.5 SD below the grade mean level of the normative sample were included in the dyslexic group. For seven dyslexics in our sample (from grade 5 and 6 ) we did not have comparative norms, as there were no standardized tests for the assessment of reading skills for Portuguese 5th and 6th graders. However, four of these seven dyslexics scored at similar level or below relative to other subjects graded 4 that were classified as 1.5 SD below the normative sample. So, we can assume that these four subjects are themselves 1.5 SD below the mean of subjects of the same grade. A re-analysis of the $\mathrm{P} 1, \mathrm{~N} 1$, and N320 effect after excluding the other three dyslexics (i.e., those whose raw scores were not below graders 4) confirmed our results including all pre-adolescent children; therefore we decided not to exclude these subjects.

The dyslexic group was matched with 20 age-matched controls, aged $9-13$ years ( 10 males and 10 females; mean age $[ \pm s t d]=10.6$ $[ \pm 1.7]$ years), who were recruited from local schools and classified by their teachers as average pupils and competent readers. All controls had intelligence scores in the normal range (Raven Colored Matrices) and reading speed scores within or above the average of the normative sample (3DM reading test). No statistical differences were observed between groups regarding age or intelligence ( $p>.2$ for both); however, as expected, these groups strongly differed in their average 3DM reading raw scores $(p<.001)$. Informed consent was obtained from all of the parents of the participants in compliance with the Helsinki Declaration.

\subsection{Stimulus material}

The stimulus type was manipulated in five experimental conditions: high and low-frequency words, which were selected from a Portuguese database, pseudowords, consonant sequences, and symbol sequences. The length of the words varied from one syllable (3 letters) to two syllables (4-5 letters), and only concrete nouns were included. Both pseudowords and consonant sequences were derived from the selected high-frequency words. Each highfrequency word was transformed into a pseudoword (phonologically legal) by changing the second syllable; the consonants were modified, and the vowels were kept (e.g., bico [beak] - bivo). For the construction of the consonant sequences (neither had phonological or semantic representations), the criterion was to keep the original words' consonants and to change the vowels using other consonants (e.g., bico [beak] - brcn). For the symbol sequences condition, twenty-one different non-orthographic symbols were selected. The symbol sequences were matched in length with the high-frequency words, and a correspondence between letters and symbols was established and kept consistent (e.g., $\square \diamond \bigcirc \diamond$ ). In total, 80 stimuli per condition were selected. 


\subsection{Experimental procedures}

In the implicit reading task, after the stimulus presentation, two alternative responses were displayed simultaneously on the screen: one letter/symbol that was presented in the previous stimulus and one letter/symbol that was not presented in the previous stimulus. The relative position of the letter/symbol in the stimuli was randomly determined. The subjects had to decide which of the two alternative responses was present in the preceding stimulus by pressing the corresponding response button (left or right shift). By asking subjects to make a decision we ensured that they were engaged and motivated throughout the entire task (which is particularly relevant as we have sampled pre-adolescent children), while also provide an index by which to assess behavioral performance. The 400 stimuli (80 for each condition) were presented in eight pseudo-randomized blocks of 50 trials each; conditions were balanced within blocks. Each stimulus was presented only once per subject during the experiment. The assignment of the items to the block, and the order of the presentation of the items within block, was random. Presentation software (version 0.7 ; nbs. neuro-bs.com/presentation) was used to display the stimuli on a computer screen and to register the subject responses. Words, pseudowords, and consonant sequences were presented in lowercase ("Arial"; font size 60; black font on white background). All stimuli were presented at eye-level at the center of the screen, $\sim 60 \mathrm{~cm}$ away from the subject, and ranged from 2.0 to $3.7^{\circ}$ of visual angle.

The trial sequence of events was as follows (Fig. 1): first, a fixation cross (+) was presented for $300 \mathrm{~ms}$ in the center of the screen; then, the stimulus was displayed for $1500 \mathrm{~ms}$, followed by the two alternative responses, which remained on the screen until the participant pressed a response button. The screen position of the correct responses was counterbalanced. The subjects were instructed to avoid blinking and moving during stimulus presentation; they were allowed to blink their eyes during the response period. After this response period, an extra time period of $2000 \mathrm{~ms}$ was given for the participants to blink their eyes (indicated by several stars $* * * *$ on the screen). The participants were encouraged to pause after each block. Before the task, the participants were given eight practice trials for each condition to adequately familiarize themselves with the experimental tasks.

\subsection{ERP recordings}

Continuous electroencephalogram (EEG) was acquired through the ActiveTwo Biosemi electrode system from $32 \mathrm{Ag} / \mathrm{AgCl}$ active

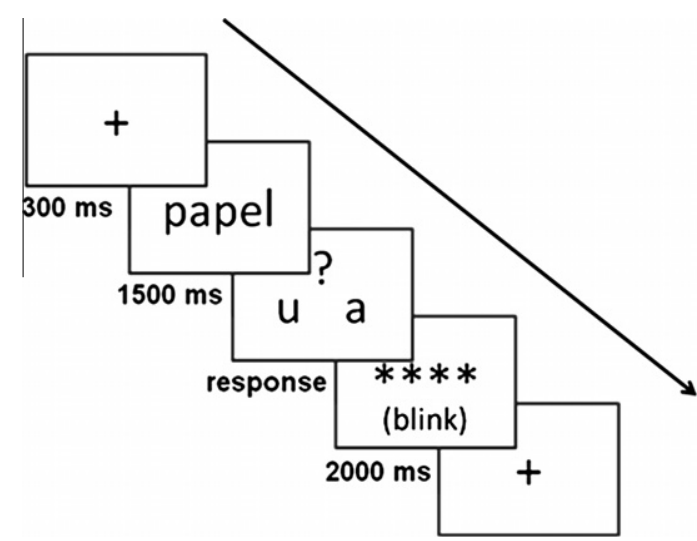

Fig. 1. Schematic illustration of the trial sequence of events. Subjects were instructed to decide which of the two alternative responses was present in the preceding stimulus and to press a corresponding response button. scalp electrodes that were mounted in an elastic cap. These electrodes were located at standard left and right hemisphere positions over the frontal, parietal, occipital, and temporal areas. They were also positioned according to the International 10-20 system guidelines (Fig. 2). Two additional electrodes were used as an online reference: Common Mode Sense (CMS) active electrode and Driven Right Leg (DRL) passive electrode. BioSemi replaces the ground electrodes that are used in conventional systems with these two electrodes. CMS/DRL form a feedback loop that drives the average voltage of the subject (i.e., the common mode voltage) as close as possible to the reference voltage of the analog-to-digital converter. Signals are recorded as the voltage between each electrode and the CMS (for a complete description, see biosemi.com; Schutter, Leitner, Kenemans, \& van Honk, 2006). Three other electrodes were attached over the right and left mastoids and below the right eye, to monitor eye movements and eye blinks. Bioelectrical signals were amplified using an ActiveTwo Biosemi amplifier (DC-100 Hz bandpass, $3 \mathrm{~dB} /$ octave) and were continuously sampled (24 bit sampling) at a rate of $512 \mathrm{~Hz}$ throughout the experiment.

\subsection{ERP data analysis}

The EEG data were analyzed using the FieldTrip open source toolbox (Oostenveld, Fries, \& Jensen, 2009; documentation and algorithms available at ru.nl/fcdonders/fieldtrip). ERP data were averaged over an epoch of $1325 \mathrm{~ms}$ (from $200 \mathrm{~ms}$ prior to until $1125 \mathrm{~ms}$ following the stimulus) and were time-locked to the onset of the stimuli. Trials containing ocular artifacts, movement artifacts, or electric noise were excluded before averaging the trials. Three dyslexic subjects and one control subject were not included in the ERP analysis due to a high percentage of artifacts (more than $30 \%$ of the trials per condition). Moreover, only trials with correct responses were analyzed. The mean (SD) number of the accepted epochs in the grand averages in normally reading and dyslexic subjects were respectively, 65.3 (4.7) and 57.4 (3.7) for consonant sequences, 61.5 (4.1) and 55.7 (3.1) for symbol sequences, 62.5 (4.0) and 62.5 (5.1) for high-frequency words, 64.6 (5.4) and 61.5 (4.7) for low-frequency words, and 66.1 (3.2) and 61.9 (4.7) for pseudowords. Corrected trials were filtered offline ( $30 \mathrm{~Hz}$ lowpass) and

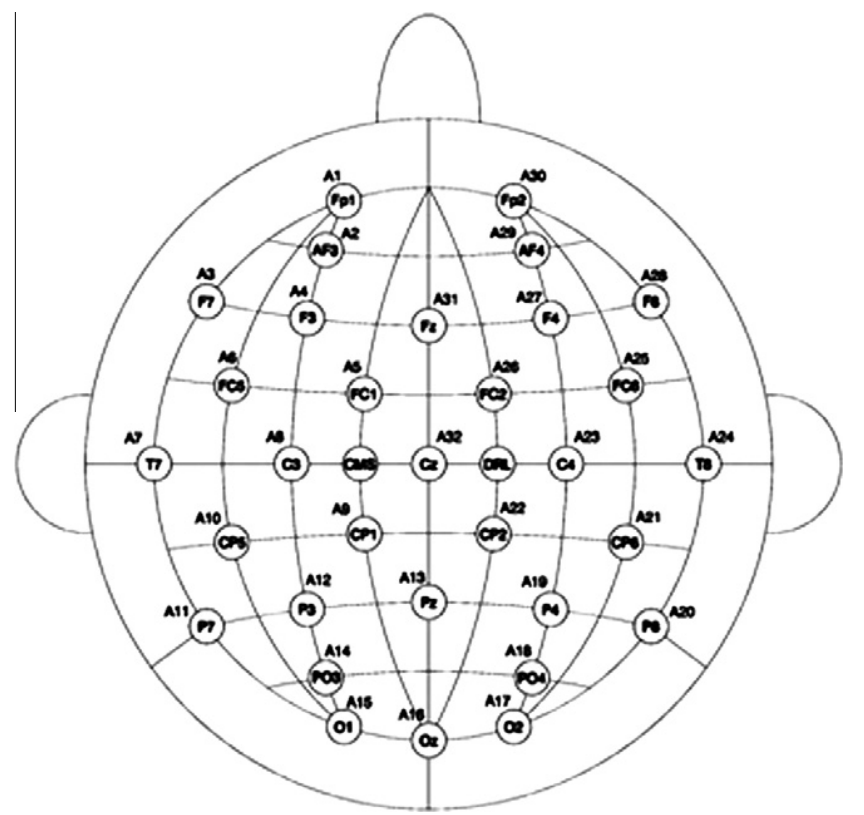

Fig. 2. The thirty-two-channel electrode montage including all standard sites in the 10-20 system. 
re-referenced to average reference (eye electrodes were excluded to compute the common reference). ERP data were analyzed by computing the mean amplitudes of the waveforms during selected time windows, relative to the $200 \mathrm{~ms}$ pre-stimulus baseline. Individual ERPs were averaged within each experimental condition and for each group. Significant ERP differences between stimulus conditions were computed separately for each group.

Three main sets of analyses were performed. To analyze the effects of letter-specific processing, we focused on the comparison of the ERPs that were elicited by the consonant sequences to the symbol sequences, during the time windows from $110 \mathrm{~ms}$ to $160 \mathrm{~ms}$ (P1 component) and from $180 \mathrm{~ms}$ to $260 \mathrm{~ms}$ (N1 component). Next, whether the P1 and N1 could reflect orthographic familiarity at the whole-word level was assessed by comparing the ERPs to familiar word-forms (i.e., high-frequency words) with the ERPs to unfamiliar word-forms (i.e., low-frequency words and pseudowords). For these comparisons, the statistical analysis of ERPs were limited to four posterior $(\mathrm{P} 3 / 4, \mathrm{P} 7 / 8, \mathrm{PO} / 4)$ and three anterior electrode sites (AF3/4, F3/4, FC5/FC6). Lastly, to assess the activity that was associated with the phonological processing, we compared pseudowords (phonologically legal) with consonant sequences (phonologically illegal) in the time window between 290 and $360 \mathrm{~ms}$ (N320 component). For the phonological analysis, the sites of interest spanned the middle and temporal areas (T7/T8, C3/C4, CP5/CP6). The selection of the time segments and electrode sites for use in specific analyses was based on visual inspection of the individual waveforms that seemed to be modulated by stimulus type. This selection also took the literature concerning the distinct levels at which words are processed into account (see for instance, Bentin et al., 1999). The statistical differences among the ERP components for the different stimulus conditions and for each group were tested with repeated measures ANOVAs, including the Stimulus Type, Electrode Site, and Hemisphere (right vs. left) as withinsubject factors and the Group as a between-subject factor. For the analysis of the effect of orthographic familiarity, Greenhouse-Geisser adjustments were employed to correct for violations of sphericity. Post-hoc analyses (Tukey HSD) were conducted to investigate significant interactions.

\section{Results}

\subsection{Behavioral results}

The data were analyzed using a repeated-measures ANOVA, with Stimulus Type as a within factor and reading Group as a between factor.

\subsubsection{Response time analysis}

Response times (RTs) from the incorrect answers and implausibly long or short RTs (i.e., 3 SD below or above the subject and condition means) were excluded from the analysis. Mean RTs were calculated for each subject and each condition. A significant main effect of Group on RTs, $[F(1,37)=6.3 ; p=.017]$, indicated that responses were generally slower for the dyslexic group $(1405 \pm 340 \mathrm{~ms})$ as compared to the age-matched controls $(1168 \pm 246 \mathrm{~ms}$; Fig. 3). Neither a significant Stimulus Type effect nor a significant two-way interaction between Group and Stimulus Type were obtained.

\subsubsection{Accuracy analysis}

A main effect of Group was observed, $[F(1,37)=27.4 ; p<.001]$. Regardless of the stimulus type, the subjects with dyslexia made relatively more errors in comparison with their respective controls, ( $81 \%$ and $95 \%$ correct answers, respectively; $p<.001$ ). A significant main effect of the Stimulus Type was also found $[F(4,15)=53.1$;

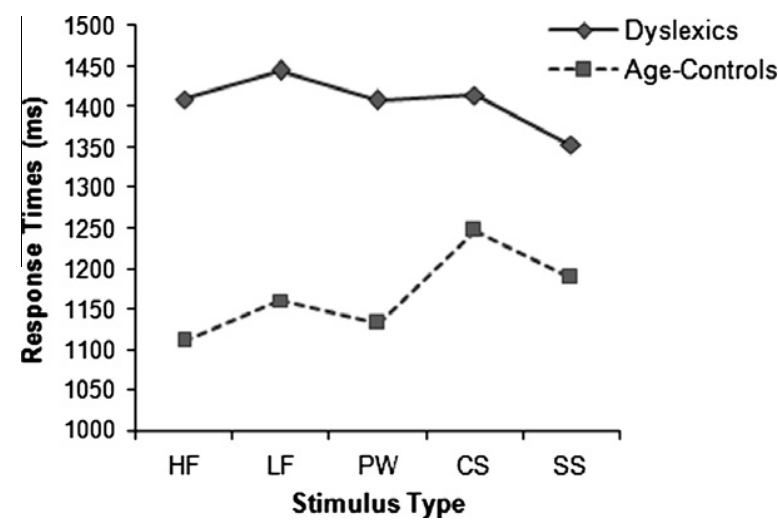

Fig. 3. Mean response times for each stimulus type (HF: high-frequency words, LF: low-frequency words, PW: pseudowords, CS: consonant sequences, SS: symbol sequences) and for each group. The error bars represent standard error of the mean.

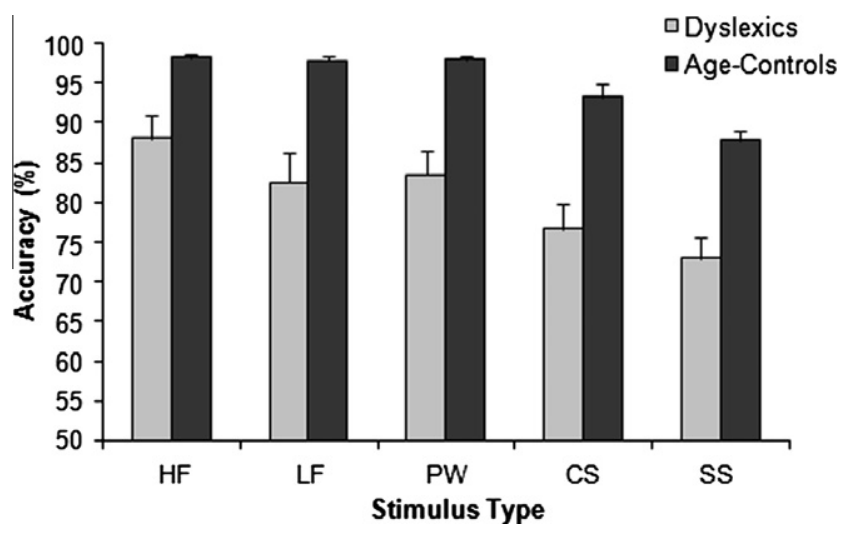

Fig. 4. Mean accuracy for each stimulus type (HF: high-frequency words, LF: lowfrequency words, PW: pseudowords, CS: consonant sequences, SS: symbol sequences) and for each group. The error bars represent standard error of the mean.

$p<.001]$. This effect was due to significantly more errors for the consonant and the symbol sequences compared to the other conditions ( $p<.001$, for all comparisons) and to higher error rates in the symbol than in the consonant sequences $(p=.020)$. Lastly, the effect of Stimulus Type on errors was modulated by the factor Group, as indicated by the interaction Group by Stimulus Type $[F(4,15)=5.2 ; p=.001]$. The controls' responses were less accurate to symbol than to consonant sequences $(p=.006)$, but the same difference failed to reach significance in dyslexics. On the other hand, while controls performed equally well in words with high and low-frequency, the dyslexics performed significantly worse on the last $(p=.009$; Fig. 4$)$.

\subsection{Electrophysiological results}

\subsubsection{Early ERP components}

3.2.1.1. Comparison of consonant sequences with symbol sequences.

3.2.1.1.1. P1 (110-160 ms). A marginally significant main Stimulus Type effect indicated that the early P1 response in the ERPs was smaller for the consonant than for the symbol sequences, $[F(1,34)=4.0, p=.054]$. The main effect of Electrode Site was also significant, $[F(1,34)=144.6, p<.001]$, as well as the interaction between Electrode Site and Hemisphere, $[F(1,34)=5.9, p=.021]$. The observed interaction was due to the fact that at posterior sites, the ERPs elicited by consonant and symbol sequences were more positive over the right hemisphere than the left hemisphere, while at anterior sites, the ERPs showed bilateral activity. Lastly, a trend for an interaction between Group and Stimulus Type indicated that 


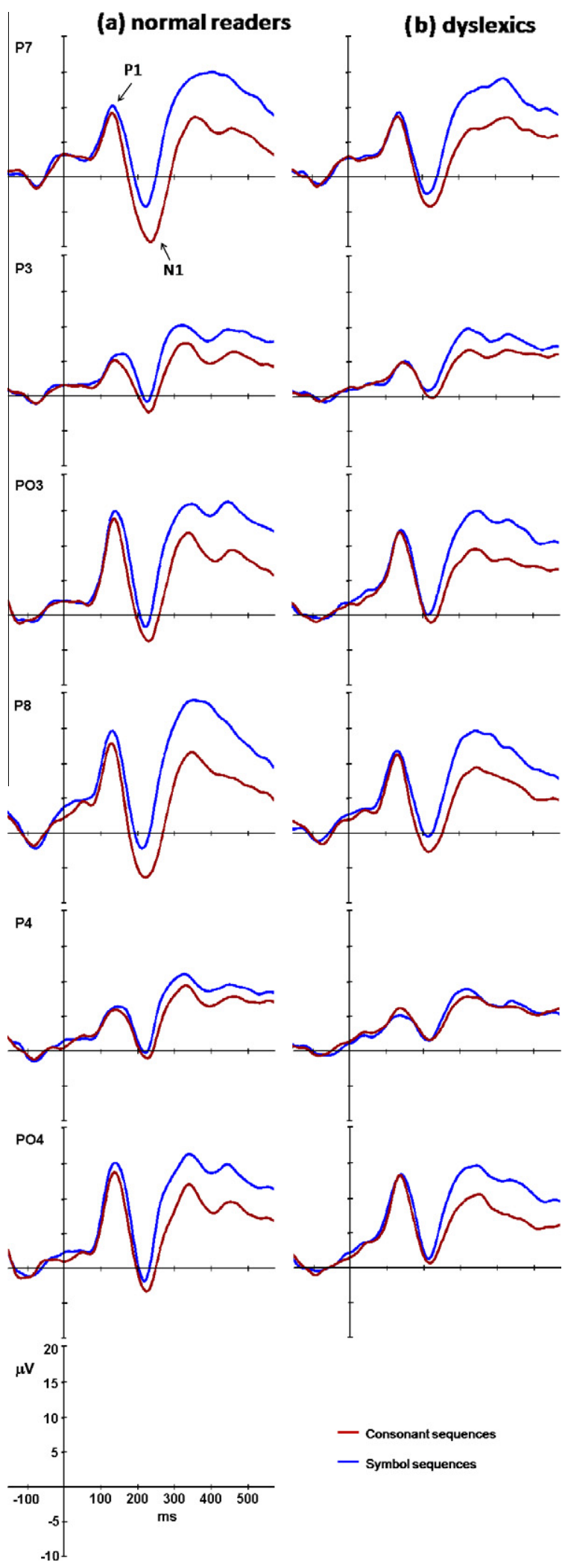

Fig. 5. ERPs elicited by consonant sequences (red line) and symbol sequences (blue line) for selected electrodes, averaged separately for (a) normal readers and (b) dyslexics. (For interpretation of the references to color in this figure legend, the reader is referred to the web version of this article.)

only the control readers differentiate processing of consonant vs. symbol sequences. $[F(1,34)=3.0, p=.094]$.
3.2.1.1.2. N1 (180-260 ms). A main effect of Stimulus Type was found, $[F(1,34)=12.5, p<.002]$, with responses to consonant sequences being more negative than the responses to symbol sequences. The interaction Stimulus Type by Electrode Site was significant, $[F(1,34)=13.8, p<.002]$, as well as the interaction Electrode Site by Hemisphere, $[F(1,34)=4.6, p=.039]$. Post-hoc analyses revealed that, at left posterior locations, the ERPs to consonant sequences were overall more negative than the ERPs to symbol sequences, while no such effect was found at anterior locations. More importantly, the Stimulus Type also interacted with Group, $[F(1,34)=4.8, p=.035]$, as the ERPs to consonant and symbol sequences were significantly different in the control group $(p<.002)$ but failed significance in the dyslexic group (Figs. 5 and 6 ). We note that similar N1 differences between the groups were found when we ran an ANOVA on the four conditions with orthographic stimuli (i.e., the mean of high and low-frequency words, consonant-sequences, and pseudowords) vs. symbol sequences. Again, this comparison showed specificity for letter-processing in control readers but not in dyslexics, as indicated by the interaction between Stimulus Type and Group, $F(1,34)=8.2, p=.007$. This analysis of variance also showed that the interaction between Stimulus Type and Electrode Site, $[F(1,34)=30.9, p<.001]$, and between Electrode Site and Hemisphere, $[F(1,34)=6.3, p=.018]$, were significant.

\subsubsection{Comparison of familiar with unfamiliar word-forms.}

3.2.1.2.1. P1 (110-160 ms). The ERPs to high-frequency words, low-frequency words, and pseudowords did not differ $(F<1)$. Additionally, no Group $[F(1,34)=1.7, p=n s]$ or interaction of Group with Stimulus Type $(F<1)$ were observed. Only interaction Electrode Site by Hemisphere was found, $[F(1,34)=4.5, p=.042]$, as posterior electrode sites elicited leftward asymmetry whereas anterior electrode sites presented bilateral activity.

3.2.1.2.2. N1 (180-260 ms). No Stimulus Type and no Group or interaction were evidenced $(F s<1)$. Only the interaction between Electrode Site and Hemisphere was found, $[F(1,34)=6.0$, $p=.020]$, revealing that, at posterior sites the $\mathrm{N} 1$ elicited by all stimuli was more negative over the left than over the right hemisphere, whereas anterior sites presented bilateral activity.

\subsubsection{Late ERP component}

3.2.2.1. Comparison of phonologically legal with phonologically illegal stimuli.

3.2.2.1.1. N320(290-360 ms). ERPs to pseudowords and consonant sequences were significantly distinct $[F(1,34)=6.1, p=.019]$, with pseudowords showing larger amplitude than the consonant sequences. A main effect of Hemisphere was also observed, $[F(1,34)=7.8, p=.009]$, as stimuli elicited significant leftward asymmetry. Interestingly, the interaction between Group and Hemisphere approached significance, $[F(1,34)=3.5, p=.059]$, reflecting the fact that in the control group, the medial and temporal sites exhibited leftward asymmetry, whereas in the dyslexic group these sites showed bilateral activity. No other main or interaction effects were observed (Fs $<1$; Figs. 6 and 7 ).

\section{Discussion}

In this ERP study, we aimed to extend previous results related to the different temporal dynamics observed in pre-adolescent children with dyslexia and normally reading controls, related to early prelexical, letter-specific processing, and late, implicit phonological processing. We used an implicit reading task and compared the ERPs associated with five types of stimuli that varied in terms of letter-specific processing, familiarity, and pronounceability. First, we hypothesized that early letter-sequence-sensitive 


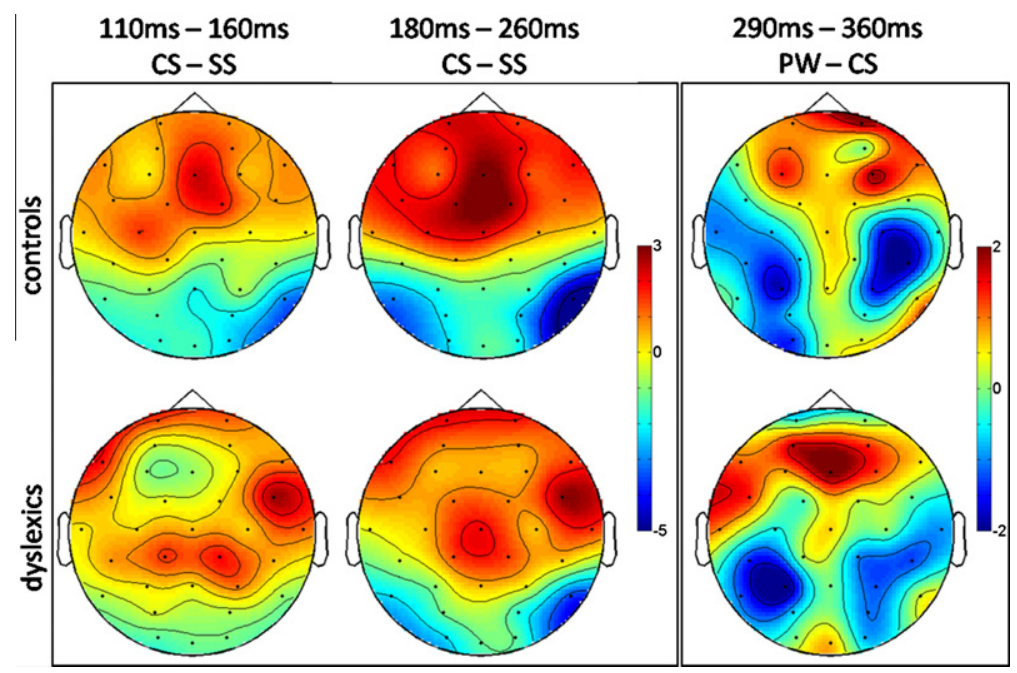

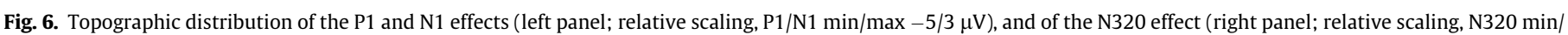
$\max -2 / 2 \mu \mathrm{V}$ ) in control and dyslexics readers.

processing observed in normally developing readers would be impaired in subjects with dyslexia. More specifically, we predicted that this would be reflected in attenuated ERP-amplitude differences between consonant and symbol sequences in the dyslexics compared to the controls.

Consistent with previous ERP findings, normally developing readers showed an early negative ERP component peaking around $200 \mathrm{~ms}$ post-stimulus onset over left posterior sites. This N1 amplitude was significantly larger for consonant compared to symbol sequences. This effect probably reflects specificity for letter-processing and is therefore compatible with the coarse print-specific N1 specialization described in the reading literature (e.g., Brem et al., 2009). This effect was also seen for the other letter sequence conditions compared to symbols in the normal readers, In addition, although the $\mathrm{N} 1$ component appeared as the strongest index of letter-specific processing, consonant and symbol sequences evoked responses already in the earlier P1 component (i.e., enhanced P1 response to symbol sequences). This has previously been found in children (e.g., Maurer et al., 2011) and suggests that letter-specific processing is under way well before $200 \mathrm{~ms}$ (Brem et al., 2006). Thus, the observed ERP activity in the P1/N1 window is consistent with the idea that the reading network specializes for visual aspects of written text during childhood (Kronbichler et al., 2006; Maurer et al., 2005, 2006; Parviainen et al., 2006). This specialization likely reflects a reorganization of the brain that occurs within a short time period as a function of reading training. Moreover, automatic skilled reading is associated with left hemispheric dominance (Maurer et al., 2005; McCandliss et al., 2003) and the left hemisphere dominance for letter sequences in our non-impaired readers thus suggests that reading in pre-adolescent children is already automated.

In the dyslexic group, the $\mathrm{N} 1$ response did not show the letterprocessing sensitivity found in the normal readers. In contrast to the control readers, the $\mathrm{N} 1$ peak was attenuated, consistent with ERP findings of attenuated P1/N1 responses to orthographic stimuli in dyslexic children (Maurer et al., 2007) and adults with severe dyslexia (Helenius et al., 1999). Paralleling these ERP findings, the performance accuracy was greater for consonant compared to symbol sequences in the controls, while this was not the case for the dyslexic participants. Taken together, the behavioral and ERP findings in the dyslexics thus provided support for an impairment in the fast, coarse processing specialization for print in developmental dyslexia (e.g., Kast, Elmer, Jancke, \& Meyer, 2010; Maurer et al., 2007; van der Mark et al., 2009). This processing specialization in non-impaired readers might represent the initial, "first pass" specialization of the VWFA. One may argue that although the dyslexic deficit is evident already during learning to read, the deficit remains, including neural markers, throughout development. One possibility is that letter-sequence-specific neuronal groups do not develop normally or that the development of these is delayed which affects the perceptual encoding of orthographic properties, therefore preventing normal development of automatic reading skills in dyslexics.

While the P1 and N1 components reflect low-level text specialization, we also tested whether these early ERP responses are sensitive to orthographic familiarity at the whole-word level. To answer this question, we analyzed lexicality and text frequency effects. This analysis of the P1 and N1 responses showed no differences between high-frequency words and low-frequency words or pseudowords, for the controls and dyslexic readers. Thus, our results suggest that for pre-adolescent children (9-13 years), the ERP responses up until about $200 \mathrm{~ms}$ post-stimulus onset to letter sequences are not sensitive to variation in orthographic familiarity. This differs from previously reported effects in the $\mathrm{N} 1$ range in adults. For instance, Hauk and Pulvermüller (2004) reported word frequency effects in a time window between 150 and $200 \mathrm{~ms}$. Hauk and colleagues (Hauk, Davis, Ford, Pulvermüller, \& Marslen-Wilson, 2006) showed larger brain activation for pseudowords than for words at about $160 \mathrm{~ms}$, whereas in Taroyan and Nicolson's study (2009) the word/pseudoword differences were found for P1 amplitude as early as $110 \mathrm{~ms}$. It has been proposed that these effects of familiarity reflect facilitation of early access to lexicosemantic information (Hauk \& Pulvermüller, 2004). In the present study, the absence of a significant amplitude difference between familiar and unfamiliar words in earlier time windows encompassing the $\mathrm{P} 1$ and $\mathrm{N} 1$ suggests that these responses might arise at the earliest prelexical stages of the orthographic system, as opposed to reflecting lexical orthographic processing. Moreover, the finding of similar $\mathrm{P} 1 / \mathrm{N} 1$ responses to words and consonant sequences strengths this interpretation, as it suggests insensitivity of the $\mathrm{P} 1 / \mathrm{N} 1$ to orthographic structure of the letter sequences (only words conform to orthographic rules). However, it is important to note that our data do not exclude the possibility that the effect of word frequency can be modulated by task demands, which would suggest that word frequency effects are more related to task-specific decision processes, rather than lexico-semantic 


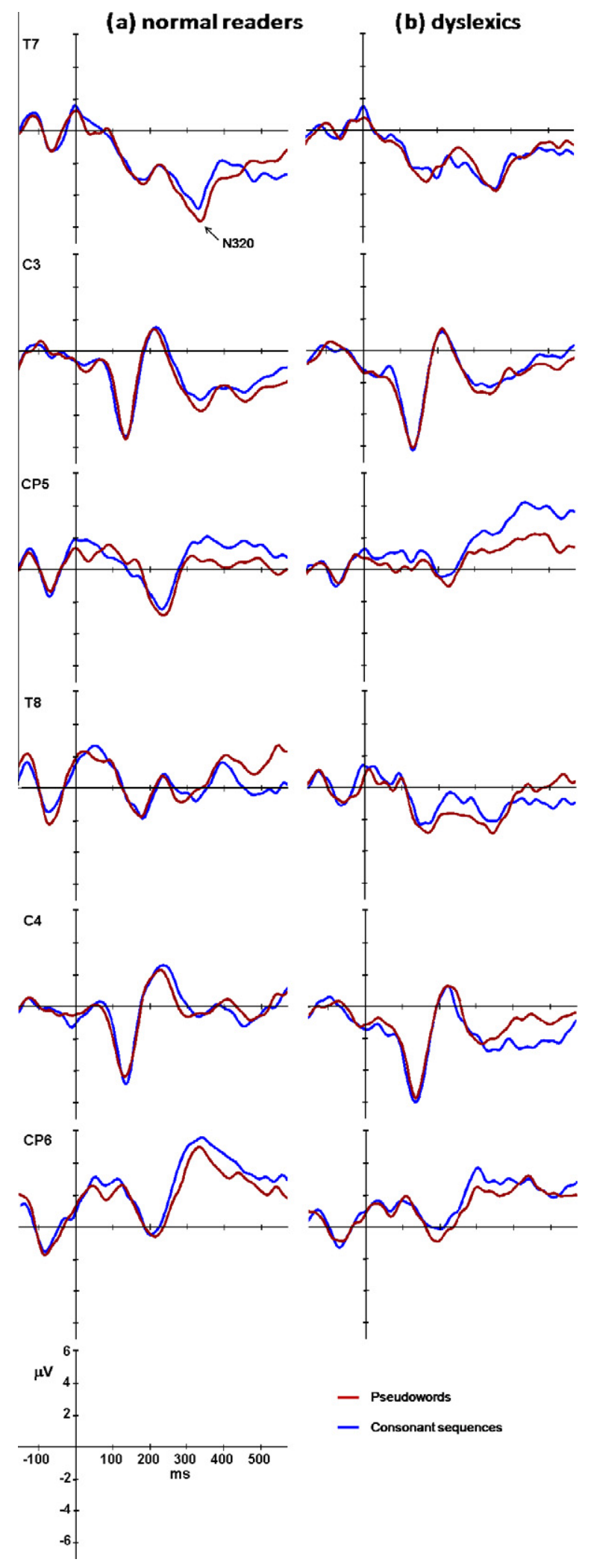

Fig. 7. ERPs elicited by pseudowords (red line) and consonant sequences (blue line) for selected electrodes, averaged separately for (a) normal readers and (b) dyslexics. (For interpretation of the references to color in this figure legend, the reader is referred to the web version of this article.)

processing (see, for example, Carreiras, Mechelli, \& Price, 2006; Chee, Hon, Caplan, Lee, \& Goh, 2002).
A second goal of the present study was to investigate ERP measures of on-line phonological processing in dyslexics, without imposing the requirements of an explicit phonological task. We addressed this issue by assessing changes in the N320 component that is believed to reflect phonological processes (Bentin et al., 1999), by contrasting phonologically legal/pronounceable (pseudowords) vs. illegal/unpronounceable (consonant sequences) stimuli. In both groups, the pseudowords triggered a larger negative amplitude than the consonant sequences did. The observed N320 modulation is consistent with similar phonological N320 effects reported in the literature (Bentin et al., 1999; Simon et al., 2004), and may be explained by intrinsic differences in the processing operations that occur; that is, the pseudowords obey phonological rules and thus can benefit from phonological recoding for maintaining stimuli in working memory (e.g., Baddeley, 1986) - note that our participants have to keep the entire letter sequences in working memory until they knew what the target letter was - whereas consonant sequences cannot significantly or systematically activate phonological information. Admittedly, an alternative interpretation of the differences found between pseudowords and consonant sequences should be considered. It is clear that consonant sequences can be discriminated from pseudowords on the basis of pronounceability, but both stimulus types can be discriminated on the basis of visual/orthographic structure as well. The pseudowords obey orthographic and phonological rules, and can therefore activate both sources of information, whereas the consonant sequence does not. Although one may reason that modulations of the N320 response are rather late to index orthographic analysis, some caution must be exercised when interpreting our finding.

As impoverished phononological processing often underlies impaired reading ability in dyslexia (e.g., Georgiewa et al., 2002; Shaywitz \& Shaywitz, 2005), we expected that dyslexics would show smaller N320 amplitudes than normal readers. However, the phonological effect in the N320 range was indistinguishable between groups, that is, dyslexics and normal readers showed the same N320 amplitude increase in responding to pseudowords. This finding might suggest that the dyslexics did not experience an increased processing effort during the phonological coding (grapheme-to-phoneme correspondence, phoneme blending) as expected. Yet, inspection of Fig. 3 shows a trend for consonant sequences to lead to longer RTs (vs. pseudowords) in control readers, suggesting that different strategies might have been recruited during the processing of the two item types. However, this was not the case for dyslexics. Our ERP results contrast with previous studies that used explicit phonological awareness tasks (e.g., auditory and visual rhyming) and reported time course differences between reading groups (Ackerman et al., 1994; McPherson \& Ackerman, 1999; McPherson, Ackerman, Holcomb, \& Dykman, 1998). The present findings therefore suggest that phonologically related ERP effects might critically depend on the explicit requirement of phonological monitoring or awareness. The present data do not exclude the possibility that a phonological deficit would have become apparent if we had used a more demanding experimental task. For example, behavioral studies have shown that dyslexic readers exhibit greater difficulties with overt pseudoword pronunciation compared to implicit decoding tasks (e.g., Stanovich \& Siegel, 1994). Functional neuroimaging studies also described smaller group differences for easier compared to harder phonological tasks (e.g., Shaywitz et al., 1998), suggesting that task difficulty is an important factor in identifying dysfunctional activation patterns. Actually, this aspect gains particular interest given the characteristics of Portuguese orthography, which is an orthography of intermediate depth (i.e., letter-sound relations are relatively regular; Seymour, Aro, \& Erskine, 2003). In these orthographies, the phonological deficits appear to be somewhat less pervasive and 
their expression more evident when task demands increase (e.g., de Jong \& van der Leij, 2003; Wimmer, 1996). Future studies in which explicit vs. implicit processes in phonological analysis is contrasted ought to clarify whether the absent phonological effect can be explained in terms of the influence of the orthographic depth of the writing system.

An interesting observation in our analysis was the topographic differences between groups: the control readers tended to show a lateralized pattern with relatively greater left than right negativity; in contrast, the dyslexic group did not show any lateralization effect. Previous studies using an illiterate population show that literacy itself might influence the functional hemispheric balance between brain structures related to reading, including the inferior parietal and temporoparietal regions (e.g., Petersson, Silva, Castro-Caldas, Ingvar, \& Reis, 2007). Therefore, it seems likely that a relationship exists between reading expertise and the hemispheric differences reported in our study. That is, our findings are indicative of a less consolidated reading network in the left hemisphere of the dyslexic pre-adolescent children. This would be in agreement with results from earlier studies of increased righthemisphere participation in phonological tasks in individuals with dyslexia (Démonet, Taylor, \& Chaix, 2004; Pugh et al., 2000b; Shaywitz et al., 1998, 2002; for a review, see Richlan et al., 2009). For example, Shaywitz and colleagues suggested that non-impaired readers show a well-functioning left-hemisphere posterior circuit (including both dorsal, temporo-parietal and ventral, occipitotemporal components) for word and pseudoword reading, while reading-disabled individuals do not, which might be compensated by increased bilateral inferior frontal activation and an increased role of right-hemisphere posterior regions in reading (Pugh et al., 2000b; Shaywitz et al., 1998). To some extent, the pattern of our behavioral results (errors and response times) is consistent with our ERP results and suggest that compensatory mechanisms might be at play (i.e., the pseudoword performance was not more impaired than the word performance in dyslexic readers).

In summary, the dyslexics showed deviant letter-specific processing effect in earlier time windows, including the P1 and N1 components, whereas phonological effects were comparable to those of normal readers in the later N320 window. In addition, the normally reading pre-adolescent children exhibited a left-lateralized N320 topography which was not present in the dyslexic group. This suggests a lack of consolidation of the left hemisphere reading network in the dyslexics.

\section{Acknowledgments}

This work was supported by the grant SFRH/BD/28488/2006, PTDC/PSI-PCO/110734/2009, and IBB/CBME, LA, FEDER/POCI 2010, from Fundação para a Ciência e Tecnologia, as well as the Swedish Dyslexia Foundation, Max Planck Institute for Psycholinguistics, Donders Institute for Brain, Cognition and Behaviour, Radboud University Nijmegen, Vetenskapsrådet, Hedlunds Stiftelse and Stockholm County Council (ALF, FoUU). The authors also thank Paula Teles from Clínica da Dislexia and Ana Guerreiro from Clínica Alvo for their help to contact dyslexic participants.

\section{References}

Ackerman, P. T., Dykman, R. A., \& Oglesby, D. M. (1994). Visual event related potentials of dyslexic children to rhyming and nonrhyming stimuli. Journal of Clinical and Experimental Neuropsychology, 16, 138-154.

Assadollahi, R., \& Pulvermüller, F. (2003). Early influences of word length and frequency: A group study using MEG. NeuroReport, 14(8), 1183-1187.

Baddeley, A. D. (1986). Working memory. UK: Clarendon Press.

Bentin, S., Mouchetant-Rostaing, Y., Giard, M. H., Echallier, J. F., \& Pernier, J. (1999). ERP manifestations of processing printed words at different psycholinguistic levels: Time course and scalp distribution. Journal of Cognitive Neuroscience, 11(3), 235-260.
Bergmann, J., \& Wimmer, H. (2008). A dual-route perspective on poor reading in a regular orthography: Evidence from phonological and orthographic lexical decisions. Cognitive Neuropsychology, 25(5), 653-676.

Blomert, L., \& Vaessen, A. (2009). Differentiaal Diagnostiek van Dyslexie; Cognitieve analyse van lezen en spellen (Dyslexia Differential Diagnosis; cognitive analysis of reading and spelling). Amsterdam: Boom test publishers $\mathrm{B}$. V.

Brem, S., Bucher, K., Halder, P., Summers, P., Dietrich, T., Martin, E., et al. (2006). Evidence for developmental changes in the visual word processing network beyond adolescence. Neurolmage, 29, 822-837.

Brem, S., Halder, P., Bucher, K., Summers, P., Martin, E., \& Brandeis, D. (2009). Tuning of the visual word processing system: Distinct developmental ERP and fMRI effects. Human Brain Mapping, 30, 1833-1844.

Bruno, J. L., Zumberge, A., Manis, F. R., Lu, Z.-L., \& Goldman, J. G. (2008). Sensitivity to orthographic familiarity in the occipito-temporal region. Neuroimage, 39, 1988-2001.

Cao, F., Bitan, T., Chou, T., Burman, D. D., \& Booth, J. R. (2006). Deficient orthographic and phonological representations in children with dyslexia revealed by brain activation patterns. Journal of Child Psychology and Psychiatry, 47(10), 1041-1050.

Carreiras, M., Mechelli, A., \& Price, C. J. (2006). Effect of word and syllable frequency on activation during lexical decision and reading aloud. Human Brain Mapping, 27(12), 963-972.

Chee, M. W., Hon, N. H., Caplan, D., Lee, H. L., \& Goh, J. (2002). Frequency of concrete words modulates prefrontal activation during semantic judgments. NeuroImage, 16(1), 259-268.

Cohen, L., Lehericy, S., Chochon, F., Lemer, C., Rivaud, S., \& Dehaene, S. (2002). Language-specific tuning of visual cortex functional properties of the visual word form area. Brain, 125, 1054-1069.

de Jong, P. F., \& van der Leij, A. (2003). Developmental changes in the manifestation of a phonological deficit in dyslexic children learning to read a regular orthography. Journal of Educational Psychology, 95(1), 22-40.

Dehaene, S. (2010). Reading in the brain: The new science of how we read. UK: Penguin Book.

Démonet, J.-F., Taylor, M. J., \& Chaix, Y. (2004). Developmental dyslexia. Lancet, 363(9419), 1451-1460.

Georgiewa, P., Rzanny, R., Gaser, C., Gerhard, U., Vieweg, U., Freesmeyer, D., et al. (2002). Phonological processing in dyslexic children: A study combining functional imaging and event related potentials. Neuroscience Letters, 318, 5-8.

Georgiewa, P., Rzanny, R., Glauche, V., Knab, R., Kaiser, W. A., \& Blanz, B. (1999). FMRI during word processing in dyslexic and normal reading children. NeuroReport, 10, 3459-3465.

Hauk, O., Davis, M. H., Ford, M., Pulvermüller, F., \& Marslen-Wilson, W. D. (2006). The time course of visual recognition as revealed by linear regression analysis of ERP data. Neuroimage, 30, 1383-1400.

Hauk, O., Davis, M. H., \& Pulvermüller, F. (2008). Modulation of brain activity by multiple lexical and word form variables in visual word recognition: A parametric fMRI study. Neurolmage, 42, 1185-1195.

Hauk, O., \& Pulvermüller, F. (2004). Effects of word length and frequency on the human event-related potential. Clinical Neurophysiology, 115, 1090-1103.

Helenius, P., Tarkiainen, A., Cornelissen, P. L., Hansen, P. C., \& Salmelin, R. (1999). Dissociation of normal feature analysis and deficient processing of letter-strings in dyslexic adults. Cerebral Cortex, 9, 476-483.

Jednoróg, K., Marchewka, A., Tacikowski, P., \& Grabowska, A. (2010). Implicit phonological and semantic processing in children with developmental dyslexia: Evidence from event-related potentials. Neuropsychologia, 48 , 2447-2457.

Kast, M., Elmer, S., Jancke, L., \& Meyer, M. (2010). ERP differences of pre-lexical processing between dyslexic and non-dyslexics children. International Journal of Psychophysiology, 77, 59-69.

Kronbichler, M., Hutzler, F., Staffen, W., Mair, A., Ladurner, G., \& Wimmer, H. (2006). Evidence for a dysfunction of left posterior reading areas in German dyslexic readers. Neuropsychologia, 44, 1822-1832.

Maisog, J. M., Einbinder, E. R., Flowers, D. L., Turkeltaub, P. E., \& Eden, G. F. (2008). A meta-analysis of functional neuroimaging studies of dyslexia. Annals of the New York Academy of Sciences, 1145, 237-259.

Marinelli, C. V., Angelelli, P., Notarnicola, A., \& Luzzatti, C. (2009). Do Italian dyslexic children use the lexical reading route efficiently? An orthographic judgment task. Reading and Writing, 22, 333-351.

Maurer, U., Brem, S., Bucher, K., \& Brandeis, D. (2005). Emerging neurophysiological specialization for letter strings. Journal of Cognitive Neuroscience, 17(10), 1532-1552.

Maurer, U., Brem, S., Bucher, K., Kranz, F., Benz, R., Steinhausen, H., et al. (2007). Impaired tuning of a fast occipito-temporal response for print in dyslexic children learning to read. Brain, 130, 3200-3210.

Maurer, U., Brem, S., Kranz, F., Bucher, K., Benz, R., Halder, P., et al. (2006). Coarse neural tuning for print peaks when children learn to read. NeuroImage, 33 , 749-758.

Maurer, U., Schulz, E., Brem, S., van der Mark, S., Bucher, K., Martin, E., et al. (2011). The development of print tuning in children with dyslexia: Evidence from longitudinal ERP data supported by fMRI. Neurolmage, 57, 714-722.

McCandliss, B. D., Cohen, L., \& Dehaene, S. (2003). The visual word form area: expertise for reading in the fusiform gyrus. Trends in Cognitive Sciences, 7 , 293-299.

McPherson, W. B., \& Ackerman, P. T. (1999). A study of reading disability using event-related brain potentials elicited during auditory alliteration judgments. Developmental Neuropsychology, 15(3), 359-378. 
McPherson, W. B., Ackerman, P. T., Holcomb, P. J., \& Dykman, R. A. (1998). Eventrelated brain potentials elicited during phonological processing differentiate subgroups of reading disabled adolescents. Brain and Language, 62, 163-185.

Oostenveld, R., Fries, P., \& Jensen, O. (2009). Fieldtrip toolbox. http://www.ru.nl/ fcdonders/fieldtrip.

Parviainen, T., Helenius, P., Poskiparta, E., Niemi, P., \& Salmelin, R. (2006). Cortical sequence of word perception in beginning readers. The Journal of Neuroscience, 26(22), 6052-6061.

Petersson, K. M., Silva, C., Castro-Caldas, A., Ingvar, M., \& Reis, A. (2007). Literacy: A cultural influence on functional left-right differences in the inferior parietal cortex. European Journal of Neuroscience, 26, 791-799.

Price, C., \& Mechelli, A. (2005). Reading and reading disturbance. Current Opinion in Neurobiology, 15, 231-238.

Pugh, K. R., Mencl, W. E., Jenner, A. R., Katz, L., Frost, S. J., Lee, J. R., et al. (2000a). Functional neuroimaging studies of reading and reading disability (developmental dyslexia). Mental Retardation Developmental Disabilities Research Reviews, 6, 207-213.

Pugh, K., Mencl, W., Shaywitz, B., Shaywitz, S., Fulbright, R., Constable, R., et al. (2000b). The angular gyrus in developmental dyslexia: task-specific differences in functional connectivity within posterior cortex. Psychological Science, 11, 51-56.

Ramus, F., Rosen, S., Dakin, S. C., Day, B. L., Castellote, J. M., White, S., et al. (2003). Theories of developmental dyslexia: Insights from a multiple case study of dyslexic adults. Brain, 126, 841-865.

Ramus, F., \& Szenkovits, G. (2008). What phonological deficit? The Quarterly Journal of Experimental Psychology, 61(1), 129-141.

Raven, J. C., Court, J. H., \& Raven, J. (1998). Coloured progressive matrices (11 ed.). Oxford, UK: Oxford Psychologist Press.

Richlan, F., Kronbichler, M., \& Wimmer, H. (2009). Functional abnormalities in the dyslexic brain: A quantitative meta-analysis of neuroimaging studies. Human Brain Mapping, 30, 3299-3308.

Rumsey, J. M., Nace, K., Donohue, B., Wise, D., Maisog, J. M., \& Andreason, P. (1997). A positron emission tomographic study of impaired word recognition and phonological processing in dyslexic men. Archives of Neurology, 54, 562-573.

Rüsseler, J., Becker, P., Johannes, S., \& Münte, T. F. (2007). Semantic, syntactic, and phonological processing of written words in adult developmental dyslexic readers: An event-related brain potential study. BMC Neuroscience, 8, 1-10.

Sandak, R., Mencl, W. E., Frost, S. J., \& Pugh, K. R. (2004). The neurobiological basis of skilled and impaired reading: Recent findings and new directions. Scientific Studies of Reading, 8(3), 273-292.

Schlaggar, B. L., \& McCandliss, B. D. (2007). Development of neural systems for reading. Annual Review of Neuroscience, 30, 475-503.

Schutter, D. J. L. G., Leitner, C., Kenemans, J. L., \& van Honk, E. J. (2006). Electrophysiological correlates of cortico-subcortical interaction: A crossfrequency spectral EEG analysis. Clinical Neurophysiology, 117, 381-387.

Sereno, S. C., Rayner, K., \& Posner, M. I. (1998). Establishing a time-line of word recognition: Evidence from eye movements and event-related potentials. NeuroReport, 9, 2195-2200.

Seymour, P. H. K., Aro, M., \& Erskine, J. M. (2003). Foundation literacy acquisition in European orthographies. British Journal of Psychology, 94, 143-174.

Shaywitz, S. (2003). Overcoming dyslexia. New York: Vintage Books.

Shaywitz, S. E., \& Shaywitz, B. A. (2005). Dyslexia (Specific reading disability). Biological Psychiatry, 57, 1301-1309.
Shaywitz, S. E., \& Shaywitz, B. A. (2008). Paying attention to reading: The neurobiology of reading and dyslexia. Development and Psychopathology, 20, 1329-1349.

Shaywitz, S. E., Shaywitz, B. A., Pugh, K. R., Fulbright, R. K., Constable, R. T., Mencl, W E., et al. (1998). Functional disruption in the organization of the brain for reading in dyslexia. Proceedings of the National Academy of Sciences, 95, 2636-2641.

Shaywitz, B. A., Shaywitz, S. E., Pugh, K. R., Fulbright, R. K., Mencl, W. E., Constable, R. T., et al. (2001). The neurobiology of dyslexia. Clinical Neuroscience Research, 1(4), 291-299.

Shaywitz, B. A., Shaywitz, S. E., Pugh, K. R., Mencl, W. E., Fulbright, R. K., Skudlarski, P., et al. (2002). Disruption of posterior brain systems for reading in children with developmental dyslexia. Biological Psychiatry, 52, 101-110.

Simon, G., Bernard, C., Largy, P., Lalonde, R., \& Rebai, M. (2004). Chronometry of visual word recognition during passive and lexical decision tasks: An ERP investigation. International Journal of Neuroscience, 114, 1401-1432.

Simos, P. G., Breier, J. I., Fletcher, J. M., Bergman, E., \& Papanicolaou, A. C. (2000). Cerebral mechanisms involved in word reading in dyslexic children: A magnetic source imaging approach. Cerebral Cortex, 10, 809-816.

Snowling, M. J. (2000). Dyslexia. UK: Blackwell Publishing.

Stanovich, K. E., \& Siegel, L. S. (1994). The phenotypic performance profile of reading-disabled children: A regression-based test of the phonological-core variable-difference model. Journal of Educational Psychology, 86, 24-53.

Tarkiainen, A., Helenius, P., Hansen, P. C., Cornelissen, P. L., \& Salmelin, R. (1999) Dynamics of letter string perception in the human occipitotemporal cortex. Brain, 122, 2119-2131.

Taroyan, N. A., \& Nicolson, R. I. (2009). Reading words and pseudowords in dislexia: ERP and behavioural tests in English-speaking adolescents. International Journal of Psychophysiology, 74, 199-208.

Tijms, J. (2004). Verbal memory and phonological processing in dyslexia. Journal of Research in Reading, 27(3), 300-310.

van der Mark, S., Bucher, K., Maurer, U., Schulz, E., Brem, S., Buckelmüller, J., et al (2009). Children with dyslexia lack multiple specializations along the visual word-form (VWF) system. Neurolmage, 47, 1940-1949.

Vellutino, F. R., \& Scanlon, D. M. (1987). Phonological coding, phonological awareness, and reading ability: Evidence from a longitudinal and experimental study. Merril-Palmer Quarterly Journal of Developmental Psychology, 33(3), 321-363.

Wagner, R. K., Torgesen, J. K., \& Rashotte, C. A. (1994). Development of readingrelated phonological processing abilities: New evidence of bidirectional causality from a latent variable longitudinal study. Developmental Psychology, 30, 73-87.

Wimmer, H. (1996). The nonword reading deficit in developmental dyslexia: Evidence from children learning to read German. Journal of Experimental Child Psychology, 61, 80-90.

Wimmer, H., Schurz, M., Sturm, D., Richlan, F., Klack, J., Kronbichler, M., et al. (2010). A dual-route perspective on poor reading in a regular orthography: An fMRI study. Cortex, 46, 1284-1298.

Ziegler, J. C., \& Jacobs, A. M. (1995). Phonological information provides early sources of constraint in the processing of letter strings. Journal of Memory and Language, 34, 567-593. 